\title{
Neuromyelitis optica spectrum disorder
}

\section{Patient experience and quality of life}

\author{
Janine Beekman, PhD, Aysha Keisler, PhD, Omar Pedraza, MPH, Masayuki Haramura, PhD, \\ Athos Gianella-Borradori, MD, Eliezer Katz, MD, John N. Ratchford, MD, Gerard Barron, BSc, \\ Lawrence J. Cook, PhD, Jacinta M. Behne, MS, Terrence F. Blaschke, MD, Terry J. Smith, MD, and \\ Michael R. Yeaman, PhD
}

Neurol Neuroimmunol Neuroinflamm 2019;6:e580. doi:10.1212/NXI.0000000000000580

\author{
Correspondence \\ Dr. Beekman \\ Janine.Beekman@ipsos.com \\ or Dr. Yeaman \\ MRYeaman@ucla.edu
}

\begin{abstract}
Objective

To gain insights into NMOSD disease impact, which may negatively affect QoL of patients, their families, and social network.
\end{abstract}

\section{Methods}

The current study used validated instruments to assess physical, emotional, and socioeconomic burden of NMOSD on QoL among 193 patients.

\section{Results}

A majority of patients reported an initial diagnosis of a disease other than NMOSD. Overall, two-thirds of patients reported NMOSD as having a strong negative impact on physical health (Short Form-36 [SF-36] score $27.1 \pm 39.1$ ), whereas emotional well-being was relatively unimpaired on average (SF-36 score 54.0 \pm 44.9$)$. A subset of patients reported having the highest category of emotional health despite worse physical health or financial burden, suggesting psychological resilience. Pain $(\mathrm{r}=0.61)$ and bowel/bladder dysfunction $(\mathrm{r}=0.41)$ imposed the greatest negative physical impact on overall QoL. In turn, ability to work correlated inversely with worsened health $(\mathrm{r}=-0.68)$. Increased pain, reduced sexual function, inability to work, and reduced QoL had greatest negative impacts on emotional well-being. Dissatisfaction with treatment options and economic burden correlated inversely with QoL.

\section{Conclusions}

Collectively, the current findings advance the understanding of physical, emotional, social, and financial tolls imposed by NMOSD. These insights offer potential ways to enhance QoL by managing pain, enhancing family and social networks, and facilitating active employment.

From the Ipsos Public Affairs (J.B., A.K., O.P.), Washington, DC; Chugai Pharmaceutical Co., Ltd. (M.H.), Chuo-ku, Tokyo, Japan; Chugai Pharma USA, Inc., (A.G.-B.), Berkeley Heights, NJ; Viela Bio (E.K., J.N.R.), 1 Medlmmune Way, Gaithersburg, MD; MedImmune Ltd. Riverside Building (G.B.), Granta Park, Cambridge, UK; Department of Pediatrics (L.J.C.), University of Utah, Salt Lake City, UT; The Guthy-Jackson Charitable Foundation (J.M.B.), Beverly Hills; Departments of Medicine and of Molecular Pharmacology (T.F.B.), Stanford University School of Medicine, Stanford, CA; Department of Ophthalmology and Visual Sciences (T.J.S.), Kellogg Eye Center and Division of Metabolism, Endocrine and Diabetes, Department of Internal Medicine, University of Michigan Medical School, Ann Arbor, MI; Department of Medicine (M.R.Y.), University of California, Los Angeles, Los Angeles; Divisions of Molecular Medicine and Infectious Diseases, Harbor-UCLA Medical Center; and Los Angeles Biomedical Research Institute at Harbor-UCLA Medical Center, Torrance, CA. 


\section{Glossary}

ANOVA $=$ analysis of variance; AQP4 = aquaporin-4; GJCF = Guthy-Jackson Charitable Foundation; MOG = myelin oligodendrocyte glycoprotein; NMO = neuromyelitis optica; NMOSD = neuromyelitis optica spectrum disorder; $\mathbf{Q o L}=$ quality of life; SF-36 = Short Form-36.

Neuromyelitis optica spectrum disorder (NMOSD) is a potentially life-threatening neuroinflammatory disease targeting the optic nerve, spinal cord, and brain. ${ }^{1-4}$ Relapses result in cumulative neurologic disabilities, are unpredictable, and are interspersed with remissions. Increased diagnostic accuracy and increased health care provider awareness have resulted in increased prevalence up to $10 / 100,000$ in some geographic regions. ${ }^{5-8}$ This estimate equates to $>15,000$ US patients and $>100,000$ cases worldwide. NMOSD disproportionately affects females (up to 7:1). ${ }^{9,10}$ Positive anti-aquaporin-4 (AQP4) antibody neuromyelitis optica (NMO-IgG) is the most common disease serotype; however, titers fail to predict disease course. ${ }^{11,12}$ Recent evidence ${ }^{13,14}$ suggests that cases positive for anti-myelin oligodendrocyte glycoprotein (MOG) antibody (MOG-IgG) are pathogenically distinct from NMOSD.

Although studies suggest therapeutic benefit, no treatment of NMOSD has been found to be safe and effective in prospective, adequately powered clinical trials. However, recent results from phase IIb and phase III trials are encouraging. ${ }^{15-17}$ In these trials, biologic therapeutics being evaluated include those targeting the complement $\mathrm{C} 5$ protein, the intereukin-6 receptor, and CD-19 protein on B cells. In addition, new and important scientific insights have recently shed light on key mechanisms underpinning NMOSD pathogenesis that may represent targets for next-generation therapeutics. ${ }^{18-21}$

By comparison, few studies have systematically examined the impact of NMOSD on quality of life (QoL) in well-characterized cohorts. ${ }^{22-25}$ Therefore, The Guthy-Jackson Charitable Foundation, Alexion Pharmaceuticals, Chugai Pharmaceutical Co., MedImmune/Viela Bio, and Ipsos Public Affairs conducted a cooperative study of NMOSD patient experience and QoL. Through an interactive survey format, patient-reported clinical, demographic, and experiential data were systematically collected from geographically dispersed patients with NMOSD across North America. The current analyses yielded novel insights that may afford potentially modifiable aspects of personal or clinical care to improve QoL in patients with NMOSD.

\section{Methods}

\section{Clinical research standards}

\section{Human subjects protection}

The study was conducted in accordance with 45 Code of Federal Regulations Part 46 and the US Department of Health and Human Services policies regarding conductance of Human Subject Research. Protocols, survey instruments (figure e-1, links.lww.com/NXI/A120), and informed consent documents were approved by a central institutional review board. Written and verbal consent/assent were obtained before enrollment.

\section{Special population compliance}

The online survey instrument was compliant with the Americans with Disabilities Act. Participants were given the option of completing the survey with assistance of a relative, friend, or caregiver if physical impairments precluded independent participation.

\section{Study goals and design}

\section{Study goals}

Goals were (1) to gain understanding of the natural history of NMOSD from a patient-reported perspective and (2) to assess NMOSD patient QoL using a rigorous survey methodology comprising standardized and NMOSD-specific QoL measures. Both goals were intended to identify how NMOSD affects patients and in so doing identify those aspects that might be modified.

\section{Study design}

This study used a cross-sectional survey design. Comparative disease data were derived from published studies, which used identical standardized measures and for which parallel demographic and QoL data sets were available.

\section{Survey themes}

The survey instrument assessed multiple disease impacts using quantitative Likert scales with dynamic ranges respective of each theme:

\section{Health-related QoL}

Three validated scales were used to assess the impact of NMOSD on health-related QoL: (1) select items from the Role-Physical and Role-Emotional subscales of the Short Form-36 (SF-36) measured the impact of NMOSD on physical and emotional health. ${ }^{26}$ Scores on the SF-36 subscales ranged from 0 to $100(100=$ highest functioning and 0 the lowest). The scale is normalized to average US individuals having a score of 50; (2) the MS QoL scale ${ }^{27}$ measured effects of pain, bowel and bladder, and sexual function; (3) the Impact of Visual Impairment Scale assessed of visual impairment affected perceived QoL. ${ }^{28}$

\section{Perceived impact of NMOSD on daily living}

Parameters were measured by (1) overall QoL (distinct from health-related QoL); (2) perceived impact on career; (3) social life; (4) personal relationships; (5) reproduction choices; (6) NMOSD-related pregnancy complications; and (7) degree to which living situation was determined by necessity. 


\section{Diagnostic experience}

Measures included date of initial diagnosis (month/year); presenting symptoms; and diagnostic history, including time between first symptoms, initial diagnosis, and NMOSD diagnosis.

\section{Treatment experience}

The following data were collected: treatment history; reason for treatment change; date of most recent treatment change; perceived effectiveness of current NMOSD treatment; concerns regarding current treatment; and outlook on future treatment.

\section{Relapse experience}

Relapses in the previous year were measured using several aspects of impact and severity, including total number and frequency of clinically-confirmed relapses; number requiring inpatient hospitalization; treatment regimens received for relapses; average duration of relapses; and frequency of emergency/urgent care for NMOSD.

\section{Health care experience}

Evolution of patient interactions with health care professionals was assessed by first presentation to a health care provider with symptoms consistent with NMOSD; initial referral to an NMOSD specialist; specialty of physician diagnosing NMOSD; factors influencing choice of current physician; frequency of scheduled clinical evaluations; and level of satisfaction with NMOSD physician/health care provider.

\section{Economic burden}

Specific financial impact of NMOSD was estimated via time spent traveling to/from medical appointments; method of transportation; need for in-home professional care; total costs and annual out-of-pocket expenses for care; financial support received; burden of monthly out-of-pocket expenses; and perceived sufficiency of health care insurance.

\section{Future uncertainty}

Future concerns of worsening of disease and unpredictable development of improved therapies were assessed.

\section{Survey translation}

Translation of the survey into Spanish involved a 2-step process. First, a native-speaking translation linguist reviewed documents against the source English file for consistency, terminology, and syntax. Next, a computer-aided translation tool (Translation Workspace XLiff Editor, v.2.49.1) ${ }^{29}$ was applied to review the instrument in contextual modules, resolving semantic ambiguity.

\section{Eligibility and enrollment}

\section{Eligibility}

Participants were recruited from an opt-in digital mailing list of 2,000 individuals in the NMO advocacy community who requested information. Those fulfilling inclusion criteria were study eligible: self-reported, established diagnosis of NMO or NMOSD $^{30,31}$ and the ability to read textual content or hear questions audibly and respond to questions.

\section{Enrollment and implementation}

Eligible subjects were consented and enrolled. The survey instrument was implemented either by telephone or via an online interface. Both modalities offered assistance through a clinical study coordinator and provided options allowing completion in 1 session or to complete over multiple sessions. Patientreported clinical data assessed are summarized in figure e-1 (links.lww.com/NXI/A120). Survey completion most commonly occurred in the patient or caregiver residence. Caregivers sometimes assisted the patient and investigator in use of the computer interface or in patient historical recall.

\section{Informatics and data security}

Study data were collected using a web-accessible electronic data capture system with access limited to qualified study personnel. Each patient data set was curated for quality, internal consistency, and completeness.

\section{Statistical analyses}

Descriptive statistics (medians or interquartile ranges for numeric variables; counts or percentages for categorical variables) were evaluated to assess cohort demographic diversity. Pairwise analysis of variance (ANOVA), $\chi^{2}$ tests, and Pearson or Spearman correlation analyses were used to assess magnitude and orientation of relationships between or among study variables. All analyses were performed in SPSS v. 25.0. ${ }^{32}$ Probability values $(p)<0.05$ and correlation values $(r)>$ or $<0.5$ were considered significant.

\section{Data availability}

Deidentified data obtained using the survey instrument used in the current study (figure-e1, links.lww.com/NXI/A120) will be made available to qualified research personnel in accordance with institutional review board policies and upon request approximately 6 months following the final publication date.

\section{Results}

\section{Cohort demographics}

\section{Sex, race, and ethnicity}

The study population was predominantly female $(\mathrm{N}=171$; $88.6 \%$ ) and comprised diverse racial/ethnic backgrounds: $71.5 \%$ Caucasian/white; 16.1\% African American/black; 6.7\% Asian (AS); 6.7\% Hispanic/Latina/o or Spanish American; 0.5\% Native American, 1.0\% Pacific Islander; and 2.6\% Other. The distribution exceeds $100 \%$ because individuals could select multiple categories. The sample was predominantly English speaking; 2 participants requested the Spanish survey. Race and ethnicity distribution was generally representative of the U.S. population, but reflected a smaller proportion of $\mathrm{HL}$ participants than expected.

\section{Education}

Twenty-eight percent reported completing a primary or high school education or general educational development (figure 1A). Twenty-one percent hold an associates or technical 
A.

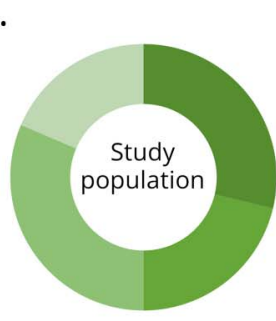

Education

- Primary/highschool/GED

associate/technical degree

Bachelor's degree

Post-graduate education

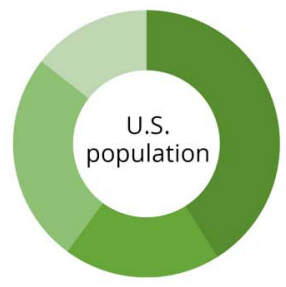

Employment
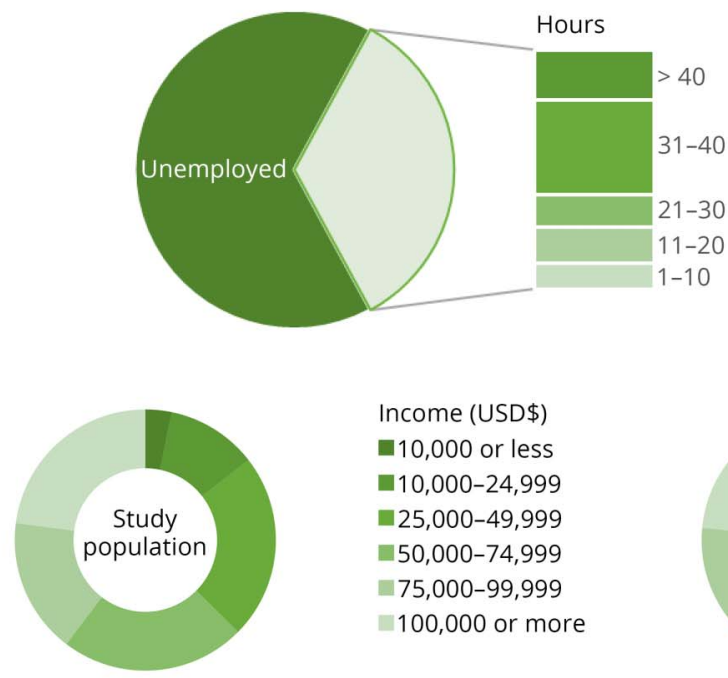

Income (USD\$)

10,000 or less

घ10,000-24,999

$=25,000-49,999$

- $50,000-74,999$

$-75,000-99,999$

100,000 or more

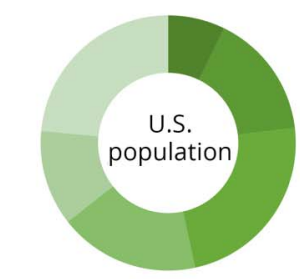

B.

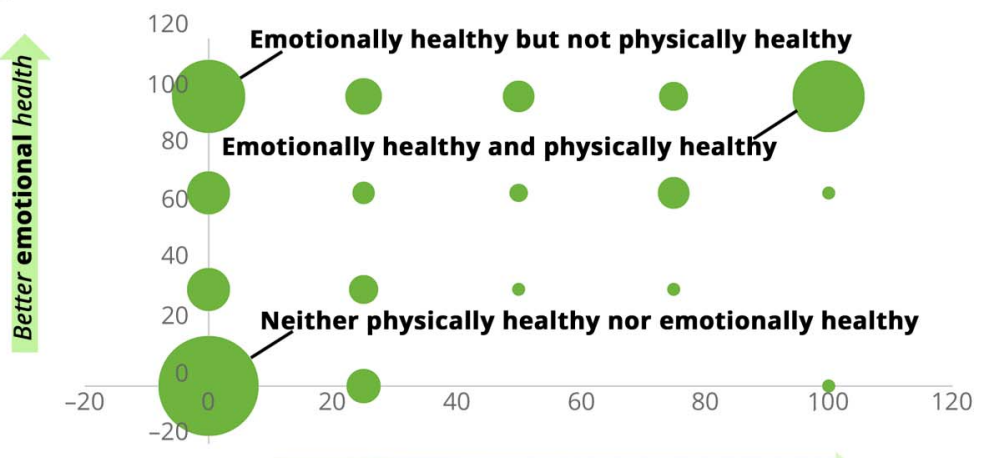

Better physical health
(B) Relationship between physical and emotional health functioning in NMO/SD. Criteria were based on SF-36 rolephysical and role-emotional health measures. The relative size of the circle represents the number of respondents with a given score. Most respondents fell within one of 3 categories, as labeled. Note that the upper left category represents a particularly resilient group of patients with very poor physical health but very robust emotional health. degree, $30.6 \%$ hold a bachelor's degree, and $18.1 \%$ have a postgraduate education or professional degree. Typical of online survey research, the sample skewed slightly to a greater proportion of subjects having a higher level of education than the general US population.

\section{Employment status}

Approximately $35 \%$ of the study cohort $(\mathrm{N}=67)$ reported current employment (figure 1A), ranging from full-time $(\geq 40 \mathrm{~h} / \mathrm{wk} ; 21.2 \%)$ to part-time work. Nine unemployed respondents (4.6\%) reported that they are actively seeking employment. Of those unemployed, 18 are full-time homemakers or caregivers, 22 are retired, and 1 is a student. Most unemployed respondents $(63.7 \% ; \mathrm{N}=79)$ reported being disabled.

\section{Income}

Household annual income varied widely among the study subjects (figure 1A). The study population comprised a smaller proportion of participants who earned less than $\$ 10,000$ per year compared with the broader US demographic.

\section{Residential status and children}

Study participants resided in one of 43 US states and the District of Columbia, whereas 11 participants resided in Canada. The modal state of residence was California $(\mathrm{N}=$ $27 ; 14.8 \%)$. The majority of participants (70.5\%) lived with their spouse/partner; $38.3 \%$ with their children; $10.4 \%$ were living alone at the time of study. None reported living with domestic assistance or in an 
institutional domicile/care facility. Most participants (73.1\%) had children.

\section{Survey assistance and future research}

Nineteen participants $(<10 \%)$ received assistance in survey completion. One participant participated by telephone. Over $92 \%$ of study subjects $(\mathrm{N}=179)$ would consider participating in a future study, whereas $8(4.1 \%)$ declined considering a future survey and $6(3.1 \%)$ declined to answer this question.

\section{Overall QoL}

\section{Physical and emotional health}

Role-physical scores were relatively low but exhibited wide variability (median $=27.1 \pm 36.1$ ). Role-emotional functioning was near-average, with broad variance $($ median $=54.0 \pm 44.9)$. Data exhibited bimodal distribution, with participants chiefly reporting either low or high functioning (figure 1B). Although physical and emotional health were positively correlated $(r=0.513$; $p<0.05)$, the data also highlighted a complex health continuum (figure 1B).

\section{Comparative QoL}

To contextualize NMOSD QoL, SF-26 data were compared with data examining other autoimmune/inflammatory disorders (table 1). Where results are summarized physically, NMOSD impact on QoL was rated similarly to systemic lupus erythematosus. Emotional impact of NMOSD was rated as

Table 1 Comparative impact and determinants of NMO/SD impact on QoL

\begin{tabular}{|c|c|c|c|c|c|c|c|}
\hline \multirow{2}{*}{$\frac{\text { Comparative }}{\text { Disease cohort }}$} & \multirow[b]{2}{*}{ Sample size } & \multicolumn{2}{|c|}{ Physical $^{a}$} & & \multicolumn{2}{|c|}{ Emotional $^{a}$} & \multirow[b]{2}{*}{ Source } \\
\hline & & $\mathbf{M}$ & SD & & M & SD & \\
\hline Current study & 193 & 27.1 & 39.1 & & 54.0 & 44.9 & - \\
\hline Other NMO ${ }^{b}$ & 30 & 36.0 & 10.7 & & 46.7 & 10.9 & Zhao et al. ${ }^{14}$ \\
\hline $\mathbf{M S}^{\mathrm{c}}$ & 368 & 18.0 & NA & & 52.0 & NA & Riazi et al. ${ }^{35}$ \\
\hline Parkinson disease $^{c}$ & 227 & 19.0 & NA & & 34.0 & NA & Riazi et al. ${ }^{35}$ \\
\hline Systemic lupus erythematosus & 1,316 & 36.3 & 41.5 & & 54.5 & 43.9 & Wolfe et al. ${ }^{36}$ \\
\hline Amyotrophic lateral sclerosis & 679 & 18.2 & 33.1 & & 47.3 & 46.2 & Jenkinson et al. ${ }^{37}$ \\
\hline Rheumatoid arthritis & 13,722 & 39.9 & 42.0 & & 63.5 & 42.4 & Wolfe et al. ${ }^{36}$ \\
\hline NI rheumatic disorders & 3,623 & 39.5 & 41.6 & & 65.6 & 41.4 & Wolfe et al. ${ }^{36}$ \\
\hline Antiphospholipid syndrome & 270 & 43.5 & 49.6 & & 56.8 & 49.4 & Georgopoulou et al. ${ }^{3}$ \\
\hline Fibromyalgia & 2,733 & 19.2 & 32.3 & & 43.9 & 43.9 & Wolfe et al. ${ }^{36}$ \\
\hline \multicolumn{3}{|l|}{ Determinant } & \multicolumn{3}{|c|}{ Range } & Mean & SD \\
\hline \multicolumn{3}{|l|}{ Overall QoL } & \multicolumn{3}{|c|}{$1-6$} & 4.58 & 1.4 \\
\hline \multicolumn{3}{|l|}{ Bodily pain } & \multicolumn{3}{|c|}{$1-6$} & 3.60 & 1.3 \\
\hline \multicolumn{3}{|l|}{ Impaired career } & \multicolumn{3}{|c|}{$1-6$} & 3.30 & 1.9 \\
\hline \multicolumn{3}{|l|}{ Ability to work at job } & \multicolumn{3}{|c|}{$1-6$} & 3.19 & 1.9 \\
\hline \multicolumn{3}{|c|}{ Affected choice whether to have children } & \multicolumn{3}{|c|}{$1-6$} & 2.11 & 1.8 \\
\hline \multicolumn{8}{|l|}{ NMO/SD-specific issue } \\
\hline \multicolumn{3}{|c|}{ Bowel/bladder function interfering with normal activities } & \multicolumn{3}{|c|}{$1-5$} & 2.26 & 1.2 \\
\hline \multicolumn{3}{|c|}{ Interfered with day-to-day work (inside or outside the home) } & \multicolumn{3}{|c|}{$1-5$} & 2.76 & 1.2 \\
\hline \multicolumn{3}{|c|}{ Satisfaction with sexual function } & \multicolumn{3}{|c|}{$1-5$} & 2.40 & 1.2 \\
\hline \multicolumn{8}{|l|}{ Social and personal relationships } \\
\hline \multicolumn{2}{|l|}{ Social life } & & \multicolumn{3}{|c|}{$1-10$} & 5.40 & 3.0 \\
\hline \multicolumn{3}{|c|}{ Personal and family relationships } & \multicolumn{3}{|c|}{$1-10$} & 5.66 & 2.8 \\
\hline
\end{tabular}

Abbreviations: MCS = Mental Health Component; NA = not available; NI = non-inflammatory; NMO = neuromyelitis optica; PCS = Physical Component Summary; QoL = quality of life; SF = Short Form.

a SF-36 scores on role-physical (physical functioning) and role-emotional (emotional functioning) of respondents diagnosed with NMO or NMOSD vs other comparison conditions of similar heterogeneity.

${ }^{b}$ Zhao et al report the SF-36 PCS score and MCS Summary Scale, a broader scale, which contains the role-physical and role-emotional subscales but captures a broader range of physical and mental/emotional functioning.

'Riazi et al. did not report SDs for subscale means. 
equivalent to MS, systemic lupus erythematosus, and antiphospholipid syndrome.

\section{NMOSD-specific experience}

\section{Impact of disease on QoL}

Specific impacts of NMOSD on QoL are summarized in table 1. On average, NMOSD imposed a significant negative effect (mean $=4.58 \pm 1.41$; scale $1-6[1=$ least impact, $6=$ greatest impact $]$ ); $>70 \%$ reported QoL to be greatly affected. Determinants most associated with negative QoL were pain, impact on career, and ability to work. Other factors were pain impairing day-to-day tasks, impact on social activities, bowel/ bladder dysfunction, and satisfaction with sexual function. Of interest, diagnosis of NMOSD failed to strongly influence the decision to have children.

\section{Initial presenting Symptom(s)}

The most common initial presenting symptoms (table 2) were numbness and/or tingling (68.4\%), difficulty walking (54.4\%), and visual disturbances (52.8\%). Other presenting symptoms are as in figure-e1 (links.lww.com/ NXI/A120).

\section{Accuracy of initial diagnosis}

Nearly two-thirds of the cohort $(\mathrm{N}=125 ; 64.8 \%)$ reported an initial diagnosis other than NMOSD. The most frequent were MS $(\mathrm{N}=80 ; 41.4 \%)$ or nonspecific optic neuritis $(\mathrm{N}=44$; $22.7 \%$ ) (table 2).

\section{Demographics and serologic status}

Participants ranged in age from 19 to 76 years (mean $=49.2 \pm$ 12.8 years), whereas $13-73$ years (mean $=44.7 \pm 12.5$ years $)$ at diagnosis and 3 months to 22 years (mean $=5.0 \pm 3.8$ years) from diagnosis to study enrollment. Eighty-two percent carry the diagnosis of NMO $(\mathrm{N}=158)$, whereas $18.1 \%(\mathrm{~N}=35)$ were diagnosed with NMOSD. Among the entire study cohort, 118 (61.1\%) reported being anti-aquaporin 4 antibody (NMO-IgG) seropositive, 41 (21.2\%) NMO-IgG seronegative, and 34 (17.6\%) did not know.

\section{Diagnostic or treatment delays}

Time from initial symptoms to correct diagnosis ranged from 0 (i.e., immediate NMO diagnosis) to 40 years (mean $=3.3 \pm$ 6.3 years). The time from correct diagnosis to treatment initiation ranged from 0 to 11 years (mean $=6$ months \pm 1.7 years). The median timespan between first symptom and correct diagnosis was 6 months, and the median interval to specific treatment initiation was 3 weeks.

\section{Perceived efficacy of current treatment}

The mean rating of perceived effectiveness of current treatment across all participants was $8.2 \pm 2.3$ on the following scale $(1-10): 10=$ treatment works very well; $1=$ treatment does not work well or at all. The most common medications were rituximab $(60.6 \%)$, prednisone/corticosteroids $(20.2 \%)$, and mycophenolate mofetil (17.1\%). Of treatments being prescribed for at least $10 \%$ of study subjects, those receiving
Table 2 Symptoms and diagnoses of initial disease episode among patients with NMO/SD

\begin{tabular}{|c|c|c|}
\hline & Count $^{a}$ & Percent $^{a}$ \\
\hline \multicolumn{3}{|l|}{ Initial symptoms } \\
\hline Numbness/tingling & 132 & 68.4 \\
\hline Difficulty walking & 105 & 54.4 \\
\hline Vision problems & 102 & 52.8 \\
\hline Pain & 95 & 49.2 \\
\hline Fatigue & 66 & 34.2 \\
\hline Bladder control problems & 51 & 26.4 \\
\hline Paralysis & 45 & 23.3 \\
\hline $\begin{array}{l}\text { Spasticity (sudden involuntary contraction of } \\
\text { a muscle) }\end{array}$ & 45 & 23.3 \\
\hline Bowel control problems & 30 & 15.5 \\
\hline Protracted vomiting & 25 & 13.0 \\
\hline $\begin{array}{l}\text { Cognitive problems (such as memory, mood, } \\
\text { and mental effectiveness) }\end{array}$ & 27 & 14.0 \\
\hline Protracted hiccups & 21 & 10.9 \\
\hline Excessive daytime sleepiness & 22 & 11.4 \\
\hline Depression & 20 & 10.4 \\
\hline Insomnia & 17 & 8.8 \\
\hline Emotional symptoms & 14 & 7.3 \\
\hline Sexual dysfunction & 10 & 5.2 \\
\hline \multicolumn{3}{|l|}{ Initial diagnoses } \\
\hline MS & 80 & 64.0 \\
\hline Optic neuritis & 44 & 35.2 \\
\hline Transverse myelitis & 37 & 29.6 \\
\hline Depression & 12 & 9.6 \\
\hline Lupus & 9 & 7.2 \\
\hline Stroke & 5 & 4.0 \\
\hline
\end{tabular}

Abbreviation: NMO = neuromyelitis optica.

a Patient may have reported more than 1 diagnosis before NMO/SD.

rituximab or mycophenolate mofetil reported highest perceived efficacy, whereas azathioprine was lowest (table 3 ). Four participants reported currently receiving no treatment, and 35 (18.1\%) reported "other" treatments.

\section{Concerns about treatment options}

More than $50 \%$ of participants $(51.8 \%)$ reported having concerns regarding their NMOSD treatment, mostly focused on future effectiveness (table 3). Eighty-eight participants (45.6\%) reported NMO medication changes over their disease course. The majority of these patients $(\mathrm{N}=48,54.5 \%)$ reported changes because of poor efficacy, whereas 32 
Table 3 Perceived effectiveness, concerns, and history of treatment among patients with NMO/SD

\begin{tabular}{|c|c|c|c|c|c|}
\hline Current treatment & Count & Percent & Rating of current treatment & SD & Range \\
\hline Rituximab & 117 & 60.6 & 8.76 & 1.88 & $1-10$ \\
\hline Prednisone/corticosteroid & 39 & 20.2 & 7.69 & 2.53 & $2-10$ \\
\hline Mycophenolate mofetil & 33 & 17.1 & 8.30 & 2.11 & $2-10$ \\
\hline Azathioprine (Imuran) & 28 & 14.5 & 7.39 & 2.36 & $2-10$ \\
\hline PLEX & 12 & 6.2 & 9.00 & 1.48 & $5-10$ \\
\hline Investigational drug/clinical trial & 3 & 1.6 & 10.00 & - & - \\
\hline Cyclophosphamide & 1 & 0.5 & 8.00 & - & - \\
\hline \multirow[t]{2}{*}{ Tocilizumab } & 1 & 0.5 & 10.00 & - & - \\
\hline & & & Count & & Percent \\
\hline \multicolumn{6}{|l|}{ Treatment concern } \\
\hline Future treatment effectiveness & & & 56 & & 56.0 \\
\hline Side effects & & & 46 & & 46.0 \\
\hline Ongoing significant disability & & & 23 & & 23.0 \\
\hline Ongoing relapses & & & 19 & & 19.0 \\
\hline Discomfort during administration & & & 13 & & 13.0 \\
\hline Inconvenience & & & 11 & & 11.0 \\
\hline Impact on pregnancy decisions & & & 7 & & 7.0 \\
\hline \multicolumn{6}{|l|}{ Treatment history } \\
\hline Azathioprine & & & 47 & & 53.4 \\
\hline Prednisone/corticosteroid & & & 47 & & 53.4 \\
\hline Mycophenolate mofetil & & & 30 & & 15.5 \\
\hline Rituximab & & & 21 & & 23.9 \\
\hline PLEX & & & 16 & & 18.2 \\
\hline IVIG & & & 6 & & 8.8 \\
\hline Cyclophosphamide & & & 5 & & 5.7 \\
\hline Investigational drug/clinical trial & & & 4 & & 4.5 \\
\hline
\end{tabular}

Abbreviations: IVIG = intravenous immunoglobulin; NMO = neuromyelitis optica; PLEX = plasma exchange.

Participants could be taking more than 1 medication; rating of current treatment captures the specific medication listed in the table and any other medications or treatments they were currently undergoing.

(36.4\%) reported intolerable side effects. Three patients (3.4\%) changed medication during pregnancy, 4 (4.5\%) participated in a clinical trial, and $10(11.4 \%)$ changed therapy because of cost (table 3).

\section{Impact of relapses}

Table 4 summarizes relapse frequency among study participants. Fifty-two patients (26.9\%) reported no relapses. Among the remaining 141 patients, 115 (81.5\%) reported relapses requiring hospitalization, whereas $26(18.5 \%)$ had relapses managed as outpatients. Forty-five participants (23.3\%) reported 6 or more relapses, with 2 patients having $\geq 6$ in the previous year. One-hundred twenty-one patients had not visited an emergency department because of relapse in the past year, whereas 6 others visited an emergency department $\geq 6$ times. Relapses were reported as lasting $<4$ weeks by 95 participants (49.2\%) (table 4); 8 patients (4.1\%) reported relapses lasting $>6$ months. Most participants who experienced relapses in the past year were treated with either IV or (44\%) or oral steroids (44\%).

\section{Health care experience}

The distribution of health care professionals initially sought by patients for care is summarized in table 5. Primary care 
Table 4 Total and annual relapse profile of study participants

\begin{tabular}{|c|c|c|c|c|c|}
\hline Relapse frequency & 0 & 1 & 2 & 3-5 & $6+$ \\
\hline \multicolumn{6}{|l|}{ Relapses ever experienced } \\
\hline Relapses experienced & 52 & 33 & 22 & 38 & 45 \\
\hline Relapses requiring hospitalization & 26 & 40 & 18 & 35 & 18 \\
\hline \multicolumn{6}{|l|}{ Relapses in the previous year } \\
\hline Relapses experienced & 85 & 34 & 7 & 9 & 2 \\
\hline Relapses requiring hospitalization & 23 & 17 & 6 & 6 & 0 \\
\hline No. of emergency department visits & 121 & 30 & 18 & 18 & 6 \\
\hline Relapse duration & & Count & & & Percent \\
\hline $1-7 d$ & & 30 & & & 22.7 \\
\hline $1-2 w k$ & & 37 & & & 28.0 \\
\hline 2-4 wk & & 28 & & & 21.2 \\
\hline $1-2 \mathrm{mo}$ & & 12 & & & 9.1 \\
\hline 3-4 mo & & 11 & & & 8.3 \\
\hline 5-6 mo & & 6 & & & 4.5 \\
\hline More than $6 \mathrm{mo}$ & & 8 & & & 6.1 \\
\hline
\end{tabular}

physicians $(\mathrm{N}=48 ; 24.9 \%)$ and general neurologists $(\mathrm{N}=$ $47 ; 24.5)$ were most commonly consulted initially, followed by emergency department physicians $(\mathrm{N}=41 ; 21.2 \%)$. The most common initial referral was to a general neurologist $(\mathrm{N}=77 ; 39.8 \%)$. Fifty-two patients $(26.9 \%)$ were referred to NMOSD specialist neurologists, $40(20.7 \%)$ to an ophthalmologist, and 26 (13.4\%) to a gastroenterologist. General neurologists were the most common specialists prescribing medications $(\sim 90.6 \%)$.

Nearly one-half of the study cohort $(\mathrm{N}=82 ; 42.4 \%)$ were examined by their doctor every 6 months, most commonly coincident with rituximab infusion. Thirty-three percent $(\mathrm{N}=$ 64) of patients saw their doctor at 3-month intervals; 16 $(8.3 \%)$ once per year, $2.6 \%(\mathrm{~N}=5)$ every month, and 6 (3.1\%) only at the time of relapse. The remainder report seeing their doctor "as needed" $(\mathrm{N}=4)$ or not at all $(\mathrm{N}=1)$.

\section{Economic burden of disease}

\section{Costs attributable to disease}

Study participants rated monthly out-of-pocket expenses due to NMOSD as $5.71 \pm 3.12$ on a 10-point scale ranging from no burden (1) to significant burden (10). As summarized in table 5 , prescription medicines accounted for the greatest portion of NMOSD medical costs for most patients $(\mathrm{N}=88$; $45.6 \%)$; many specified rituximab infusions as the single largest cost. The sole factor predictive of financial burden was receiving plasma exchange therapy during relapse. Twenty-five (13\%) reported travel costs to/from health care providers as accounting for the greatest cost, whereas 18 (9\%) stated hospitalization accounted for greatest cost. Eleven percent $(\mathrm{N}=21)$ reported that an in-home professional caregiver provides service. Other significant costs reported were herbal supplements, psychologist visits, or medical costs not covered by Medicare.

\section{Total costs}

Beyond subjective financial burden of disease, the total annual expenses reported by the cohort as a whole was $\$ 1,109,357$ or an average of $\$ 5,748$ per respondent (table 5). The most frequently reported cost was prescription medication, with 140 participants $(73.5 \%)$ reporting an average out-of-pocket cost of $\$ 1,876$ annually. Unprompted (not an original category of out-of-pocket costs), 3 participants reported lost income at an average of $\$ 65,000$ annually. Although not a categorical option, 23 respondents reported paying for specialists (including psychologists) out-of-pocket, at an average of $\$ 1,554$ annually. A large number of participants $(\mathrm{N}=$ $124 ; 64.2 \%$ ) reported travel costs to medical appointments, at an average of $\$ 468$ per respondent. The largest total cost outof-pocket was for hospitalization, accounting for $\$ 304,410$ annually in the sample, or an average of $\$ 7,248$ per respondent $(\mathrm{N}=42 ; 21.8 \%)$. Caregiver or support was another high cost, accounting for $\$ 70,580$ annually in the sample, or an average of $\$ 3,361$ per respondent $(\mathrm{N}=21 ; 10.9 \%)$.

\section{Financial support for care}

The majority of study participants $(\mathrm{N}=142 ; 73.6 \%)$ reported that health insurance sufficiently covered prescribed NMOSD medicines. Among those with insufficient health insurance, expensive copayment and insurer denials were common 
Table 5 Health care professionals encountered and annual expenses due to NMO/SD

\begin{tabular}{|c|c|c|c|c|c|c|c|}
\hline \multirow{2}{*}{$\begin{array}{l}\text { Health care } \\
\text { professional }\end{array}$} & \multicolumn{2}{|c|}{$\begin{array}{l}\text { Physician first to evaluate } \\
\text { symptoms }\end{array}$} & \multicolumn{2}{|c|}{$\begin{array}{l}\text { Specialist first to } \\
\text { receive referral }\end{array}$} & $\begin{array}{l}\text { Physician who } \\
\text { diagnosed NMO/SD }\end{array}$ & \multicolumn{2}{|c|}{$\begin{array}{l}\text { Physician currently prescribing } \\
\text { medications }\end{array}$} \\
\hline & \multicolumn{2}{|l|}{47} & \multicolumn{2}{|l|}{77} & 99 & \multicolumn{2}{|l|}{175} \\
\hline $\begin{array}{l}\text { Neurologist (NMO } \\
\text { specialist) }\end{array}$ & \multicolumn{2}{|l|}{24} & \multicolumn{2}{|l|}{52} & 83 & \multicolumn{2}{|l|}{-} \\
\hline Ophthalmologist & \multicolumn{2}{|l|}{21} & \multicolumn{2}{|l|}{40} & 11 & \multicolumn{2}{|l|}{0} \\
\hline Neuro-ophthalmologist & \multicolumn{2}{|l|}{1} & \multicolumn{2}{|l|}{3} & 3 & \multicolumn{2}{|l|}{0} \\
\hline Rheumatologist & \multicolumn{2}{|l|}{4} & \multicolumn{2}{|l|}{5} & 0 & \multicolumn{2}{|l|}{1} \\
\hline Gastroenterologist & \multicolumn{2}{|l|}{1} & \multicolumn{2}{|l|}{26} & 16 & 0 & \\
\hline Orthopedist & 1 & & 3 & & 0 & 0 & \\
\hline MS specialist & 0 & & 4 & & 4 & 5 & \\
\hline Primary care physician & 48 & & 1 & & 0 & 11 & \\
\hline $\begin{array}{l}\text { Emergency department } \\
\text { physician }\end{array}$ & 41 & & 1 & & 1 & 0 & \\
\hline Hematologist & 0 & & 1 & & 0 & 13 & \\
\hline Physiatrist & 0 & & 0 & & 0 & 1 & \\
\hline Other & 5 & & 8 & & 5 & 4 & \\
\hline Out-of-pock expenses & & $\mathbf{N}$ & & Minimum & Ma & num & Median \\
\hline Prescription medicine(s) & & 140 & & $\$ 25$ & $\$ 30$ & & $\$ 540$ \\
\hline Travel to clinical care & & 124 & & $\$ 10$ & $\$ 10$ & & $\$ 115$ \\
\hline Emergency/urgent care & & 48 & & $\$ 50$ & $\$ 15$ & & $\$ 275$ \\
\hline Medical supplies & & 52 & & $\$ 50$ & $\$ 3,0$ & & $\$ 330$ \\
\hline Hospitalization & & 42 & & $\$ 100$ & $\$ 15$ & & $\$ 1,950$ \\
\hline Caregiver or service & & 21 & & $\$ 50$ & $\$ 14$ & & $\$ 1,500$ \\
\hline Support groups & & 7 & & $\$ 8$ & $\$ 36$ & & $\$ 100$ \\
\hline Other costs & & 58 & & $\$ 80$ & $\$ 12$ & & $\$ 1700$ \\
\hline Category of expense $^{a}$ & & $\begin{array}{l}\text { Total cost } t \\
\text { respondent }\end{array}$ & sample & $\begin{array}{l}\text { No. of } \\
\text { cost }\end{array}$ & respondents reporting & $\begin{array}{l}\text { Average } \\
\text { respond }\end{array}$ & \\
\hline $\begin{array}{l}\text { Prescription medicine(s) } \\
\text { infusions) }\end{array}$ & ncluding & $\$ 262,598$ & & 140 & & $\$ 1,876$ & \\
\hline Emergency/urgent care & & $\$ 61,275$ & & 48 & & $\$ 1,277$ & \\
\hline Hospitalization & & $\$ 304,410$ & & 42 & & $\$ 7,248$ & \\
\hline Travel costs for medical & & $\$ 58,003$ & & 124 & & $\$ 468$ & \\
\hline Caregiver or support ser & & $\$ 70,580$ & & 21 & & $\$ 3,361$ & \\
\hline Medical supplies & & $\$ 34,173$ & & 52 & & $\$ 657$ & \\
\hline Support group & & $\$ 1,838$ & & 7 & & $\$ 263$ & \\
\hline Supplements & & $\$ 1,000$ & & 1 & & $\$ 1,000$ & \\
\hline Specialists & & $\$ 35,750$ & & 23 & & $\$ 1,554$ & \\
\hline Lost income & & $\$ 195,000$ & & 3 & & $\$ 65,000$ & \\
\hline Health insurance deduct & & $\$ 23,330$ & & 9 & & $\$ 2,592$ & \\
\hline Other costs (unspecified) & & $\$ 61,400$ & & 4 & & $\$ 15,350$ & \\
\hline
\end{tabular}


Table 5 Health care professionals encountered and annual expenses due to NMO/SD (continued)

\begin{tabular}{llll}
\hline Category of expense $^{\mathbf{a}}$ & $\begin{array}{l}\text { Total cost to sample } \\
\text { respondents }\end{array}$ & $\begin{array}{l}\text { No. of respondents reporting } \\
\text { cost }\end{array}$ & $\begin{array}{l}\text { Average annual cost per } \\
\text { respondent }\end{array}$ \\
\hline Collective sample & $\$ 1,109,357$ & 193 & $\$ 5,748$ \\
\hline
\end{tabular}

Abbreviations: NMO = neuromyelitis optica; QoL = quality of life.

a For each cost category, respondents rated the largest burden on QoL and estimated annual expense for each. Costs were totaled by category and averaged for cost-per-respondent and cost-in-sample estimates. Note: Other costs category included specialists, estimated lost income, health insurance deductibles, and unspecified costs.

reasons. Twenty-four study participants $(12.6 \%)$ reported receiving financial support for their NMOSD treatment, largely in the form of disability insurance, clinical trial participation, or support from friends and family.

\section{Correlation analysis}

To examine predictors of overall QoL, ANOVA was used to detect correlates among individual factors, including time since diagnosis, total relapse number (a surrogate of disease severity), and current treatments (overall and specifically for relapses). Neither time since diagnosis nor current treatment regimen was predictive of overall $\mathrm{QoL}$; however, the number of relapses correlated significantly with overall $\mathrm{QoL}$ $(p=0.001)$, with greater numbers of relapses diminishing QoL.

Other potential correlates affecting QoL in NMOSD were explored using a matrix Pearson or Spearman correlation analysis of primary data elements. As shown in table 6, multiple correlations were identified as trending to positively or negatively affecting QoL.

\section{Discussion}

The primary goal of this study was to determine the impact and correlates of NMO on patient QoL in a standardized manner using validated measures of physical and emotional health impact on daily activities potentially affecting QoL. By examining specific tangible domains of QoL in parallel to perceived overall QoL, the patient experience regarding how this rare disease affects daily life was revealed.

Several important themes were identified among the current study cohort. First, NMOSD typically has strong negative effects on physical functioning. Physical functioning was

Table 6 Exploratory correlation analyses among study data elements $\dagger$

Data Element

\begin{tabular}{|c|c|c|c|c|c|c|c|c|c|c|c|c|c|c|c|c|c|c|c|c|c|c|c|c|c|c|c|c|c|}
\hline & 1 & 2 & 3 & 4 & 5 & 6 & 7 & 8 & 9 & 10 & 11 & 12 & 13 & 14 & 15 & 16 & 17 & 18 & 19 & 20 & 21 & 22 & 23 & 24 & 25 & 26 & 27 & 28 & 29 \\
\hline 1. Age & .. & -. & -. & -. & .. & .. & -. & -. & - & - & .. & .. & - & -. & -. & -. & -. & -. & .. & -. & -. & -. & .. & -. & -. & - & - & - & -. \\
\hline 2. Serostatus $=$ Seropositive & .308 & .. & -. & .. & .. & -. & - & -. & .. & - & .. & .. & -. & .. & -. & -. & .. & .. & .. & .. & .. & -. & .. & -. & .. & - & - & .. & -. \\
\hline 3. Serostatus $=$ Seronegative & .261 & .651 & .. & -. & .. & -. & .. & - & -. & - & .. & -. & - & -. & -. & .. & -. & -. & -. & -. & .. & -. & -. & -. & -. & -. & - & -. & -. \\
\hline 4. Serostatus = Unknown & -.113 & .580 & -.240 & -. & .. & -. & - & -. & -. & -. & .. & -. & - & -. & -. & -. & .. & .. & -. & -. & -. & -. & -. & -. & - & -. & - & - & - \\
\hline 5. Physical Health Functioning & .186 & -.088 & -0.017 & .131 & .. & .. & .. & .- & .- & -. & .. & .. & -. & .- & .. & .. & .. & .. & .. & -. & .. & .- & .. & .- & .. & - & - & .. & .. \\
\hline 6. Emotional Health Functioning & .022 & .017 & .039 & -064 & .453 & - & - & -. & -. & - & -. & -. & - & -. & -. & -. & -. & -. & -. & - & - & -. & -. & -. & - & - & - & -. & - \\
\hline 7. QOL: Overall Quality of Life & .221 & .134 & -.102 & -.063 & -.482 &. .355 & - & -. & -. & - & -- & -. & - & -. & -. & -. & -. & -. & -. & - & -. & -. & -. & -. & - & - & - & -. & -. \\
\hline 8. QOL: Affect ability to work & .075 & .005 & .129 & -.134 & .075 & -444 & .543 & -- & - & - & -. & -. & - & -- & - & - & - & -. & -. & -. & -. & - & -. & -. & - & - & - & - & - \\
\hline 9. QOL: Bodily pain & .214 & .018 & .046 & .026 &. .501 &. .423 & .605 & .487 & .. & -. & .. & .. & - & -. & -. & .. & .. & . & .. & .. & . & .. & -. & .. & .. & -. & -. & -. & - \\
\hline 10. QOL: Bowel \& Bladder & .209 & .018 & .016 & .040 & .337 & -194 & .407 & .279 & .335 & - & .. & -. & -. & -. & -. & .. & -. & .. & -. & -. & -. & -. & -. & -. & -. & - & - & -. & -. \\
\hline 11. QOL: Sexual Function & .118 & .018 & .045 & -024 & .143 & .358 &,- 320 & -.194 & .215 & .203 & .. & .- & - & -. & - & -. & -. & -. & -. & - & .. & -. & -. & -. & - & - & - & - & - \\
\hline 12. QOL: Children &. .515 &., 114 & .159 & -.023 & .160 & .064 &. .120 & .048 &. .117 & -161 & .047 & .. & -. & -. & .. & -. & .. & .. & .. & .. & .. & .. & .. & .. & -. & -. & -. & -. & .. \\
\hline 13. Social and Family Life & .042 & .069 & .029 & .056 & -.170 & .247 & .204 & .436 & .193 & .180 & -191 & .023 & -- & -. & -. & -. & -. & -. & -. & -. & - & -. & -. & -. & -. & -. & - & -. & - \\
\hline 14. Future Uncertainty & .212 & .043 & .041 & -100 & .248 & -105 & .277 & .371 & .158 & .136 &. .192 & -.054 & .064 & -- & - & - & - & - & - & - & - & - & - & -. & - & - & - & - & -. \\
\hline 15. Symptom: Spinal & .044 & .002 & .050 & .057 & .051 & .070 & -.002 & .144 & .177 & .212 & .002 & .005 & .128 & .079 & .. & .. & .. & .. & .. & .. & .. & .. & .. & .. & -. & .. & - & .. & .- \\
\hline 16. Symptom: Optic & .029 & .035 & .085 & -.135 & .005 & -074 & .086 & -189 & .029 & -.011 & -.113 & .047 & .023 & .026 & -.365 & -. & -. & - & -. & - & - & -. & -. & -. & -. & -- & - & -. & - \\
\hline 17. Symptom: Nonspecific & .509 & .188 & -.063 & .173 & .070 & -.174 & -.041 & .136 & .138 & .060 & -.060 & .090 & .077 & .032 & .408 & .143 & - & -. & -. & - & - & - & -. & -. & - & - & - & - & - \\
\hline 18. Symptom: Area Postrema &. .163 & .117 & -.106 & -036 & -016 & .040 & 99 & .047 & .057 & .020 & -.026 & .142 & .033 & .065 & .119 & .153 & .069 & -. & -. & -. & -. & -. & -. & -. & -. & -- & - & - & -. \\
\hline 19. Symptom: Cerebral & .021 & .046 & .027 & .030 & -.152 & -193 & .138 & .007 & .228 & .165 & -.066 & .011 & .130 & .008 & .184 & .231 & .271 & .173 & .. & -. & .. & -. & -. & .- & - & -. & -- & -. & -. \\
\hline 20. Symptom: Other & .016 & -033 & .067 & -029 & -.153 & .004 & .007 & .112 & .039 & .031 & -.121 & -.083 & .017 &. .175 & .050 & .178 & .019 & .008 & -064 & - & -- & - & -. & -. & - & - & - & - & - \\
\hline 21. Tx: NS Non-medicinal & .033 & .129 & .038 & -.124 & .099 & -.131 & .060 & .006 & .127 & .045 & -.123 & .222 & .032 & .001 & .097 & .036 & .137 & .007 & .049 & -.122 & .. & -. & -. & -. & -. & - & - & -. & -. \\
\hline 22. Tx: NS Immune-suppressing & .050 & .035 & .047 & .006 & -056 & .072 & .127 & .148 & .058 & .004 & .013 & .034 & .081 & .039 & .096 & .138 & .086 & .035 & .038 & .038 & .027 & .. & .. & .. & -. & .. & -. & .. & -. \\
\hline 23. TX: Specific Immune-Suppressing & -106 & -148 & .149 & .029 & -019 & .015 & .008 & -.024 & .032 & .039 & -.025 & .019 & .007 & .050 & .079 & 126 & .045 & .083 & -.051 & .047 & .084 & .533 & - & -. & - & - & - & - & - \\
\hline 24. Tx: NS Immune-neutralizing & .006 & -.023 & .072 & -.047 & .014 & .010 & .029 & .001 & .008 & .044 & -.033 & .066 & .085 & .089 & .056 & .006 & .020 & .042 & .041 & .046 & .177 & .013 & .025 & - & - & - & - & - & -. \\
\hline 25. Tx: Other & .058 & .034 & .094 & .057 & .019 & .086 & .033 & .062 & .056 & .070 & -011 & .074 & -.041 & .027 & .034 & .053 & .047 & .010 & .028 & .103 & .015 & .174 & -.188 & .109 & -. & - & - & -. & - \\
\hline 26. Tx: None & .068 & -.101 & .083 & .040 & .008 & .021 & .038 & -.074 & .054 & .062 & .003 & .018 & .104 & .043 & .011 & .012 & .041 & .086 & .141 & .044 & -.056 &. .183 & .264 & -.021 & .082 & - & - & -. & -. \\
\hline 27. Polypharmacy & .005 & .068 & .089 & -009 & -084 & -056 & .136 & .091 & .135 & -.031 & -.021 & .026 & .010 & .029 & -.008 & .028 & .049 & .006 & .015 & .033 & .377 & .427 & .174 & .180 & .519 & -.118 & - & - & -. \\
\hline 28. Treatment Rating & .022 & .038 & .086 & .040 & .162 & .215 & -.314 & -.145 & .269 & .201 & .132 & .009 & .033 & .103 & .019 & .109 & .012 & .012 & .139 & .093 & .041 & .199 & .303 & .107 &. .128 &. .142 & .004 & -. & -. \\
\hline 29. Concern About Treatment & .052 & .038 & .001 & .049 & -162 & .064 & .092 & .029 & .008 & .067 & -.054 & .061 & .057 & .230 & -.074 & .035 & .103 & .069 & .104 & .063 & .050 & -010 & .060 & .098 & .042 & .019 & .022 & -315 & -. \\
\hline 30. Financial Burden & .046 & -175 & .182 & .027 & .229 & 216 & .216 & .147 & .287 & .171 & -053 & .016 & .075 & .218 & .013 & .025 & .038 & -.151 & .160 & .075 & .088 & .049 & .080 & .127 & .064 & .038 & .075 & -.067 & .082 \\
\hline
\end{tabular}


lower in the study cohort than in the general population and on par with individuals having MS or systemic lupus erythematosus. ${ }^{33-36}$ The predominant physical issues affecting QoL were bodily pain, bowel and bladder dysfunction, and visual impairment. These factors inversely correlated with ability to work, the limitation of which negatively affected QoL. Age was positively associated with many QoL measures such as pain, suggesting that disease increasingly negatively affctes QoL over time. Worse physical functioning also correlated with greater uncertainty about the future. Notably, anti-AQP4 antibody serostatus reported as negative or unknown correlated with less impact on QoL than detectable anti-AQP4 antibody. This relationship is similar to that often observed in AQP4 antibody-positive and MOG antibodypositive phenotypes. ${ }^{11-14}$ Conversely, seronegative status carried a significantly higher financial burden.

By comparison, emotional health was in general unimpaired, suggesting that poor physical health does not necessarily correspond to diminished emotional health. Although some study participants exhibited congruent emotional and physical health, a subset of participants reported the highest level of emotional health despite seriously impaired physical health. This inverse relationship suggests a degree of psychological resilience in some patients despite physical impairment. Likewise, a portion of participants reported that their disease had a positive effect on their social relationships. One possible explanation for such positive impact is that their disease provoked support network involvement. These findings are consistent with the concept and impact of psychological resilience, ${ }^{38}$ which can translate to effective personal strategies of coping with health-related challenges. ${ }^{39}$

The constellation of presenting symptoms in many patients resulted in an initial diagnosis of MS. Inaccurate diagnosis combined with delay of appropriate therapy can negatively affect long-term outcomes in NMOSD. ${ }^{40}$ However, recent implementation of international consensus criteria ${ }^{31}$ has increased the timeliness and accuracy of diagnosis and should improve care in early disease. ${ }^{41}$ The number, duration, and severity of relapses varied widely across the study cohort. This observation corresponds to the absence of a standardized definition and diagnostic algorithm for differentiating bona fide relapses from unrelated symptoms.

Not surprisingly, participants reporting higher treatment ratings also experienced higher physical and emotional functioning and higher QoL. Similarly, worse functioning was associated with larger financial burden. These themes are concordant with those of previous studies. ${ }^{22,23,41}$ Of interest, patients receiving nonspecific immune-suppressing treatments tended to rate their regimens more negatively, whereas those on target-specific treatments (e.g., biologics) rated their treatments more positively. Impact of NMO on QoL extended beyond physical and emotional costs; respondents reported a high financial burden, particularly for prescription medicines, travel costs, hospitalization, and specialist care. Furthermore, the per-respondent cost and total cost estimates in this study provide a useful estimate of personal and health care costs of NMOSD to society.

Results of the current study emphasize the significant negative impact NMOSD can have on patient QoL, particularly in relation to physical disability, pain, bowel and bladder dysfunction, or visual impairment. ${ }^{42-46}$ These manifestations correspond to reduced ability to work at a job or perform daily activities, and a decreased QoL, which also reconcile with negative impacts of anxiety, disability, or depression in NMOSD. ${ }^{25,47}$ Factors contributing to these adverse outcomes may include (1) delayed or inappropriate treatment due to initial misdiagnosis; (2) real or perceived efficacy or lack of efficacy of current treatment options; (3) lack of a standard definition of relapse; and (4) disease-specific economic burden. These issues underscore the importance of recent advances in diagnostic timeliness and accuracy, as well as ongoing clinical trials intended to establish the first approved therapies for NMOSD. Prospectively, global collaboration aimed at implementation of a standard relapse definition and severity score should contribute to improved clinical care. Likewise, the pursuit of predictive biomarkers of relapse to allow mitigating interventions and the initiation of studies aimed to durably restore immune tolerance as a curative therapy hold promise for increasingly effective medical solutions for NMOSD patients. Synergistic and prospective approaches such as these aimed at addressing disease causes and effects hold great promise to significantly add to the QoL for patients with NMOSD, other patients with rare disease, and beyond. ${ }^{48,49}$

\section{Acknowledgment}

The authors are deeply grateful to the patients who volunteered to participate in this research. Alexion Pharmaceuticals provided input into the study design and courtesy review of the manuscript. Special appreciation is expressed to Ms. Megan Weber for analytic support. This collaborative project was supported in-part by The Guthy-Jackson Charitable Foundation, Alexion Pharmaceuticals, Inc., Chugai Pharmaceuticals Co., Ltd., Viela Bio, and MedImmune, Ltd.

\section{Study funding}

This study was sponsored in part by The Guthy-Jackson Charitable Foundation, Alexion Pharmaceuticals, Inc., Chugai Pharmaceutical Co., Ltd., Viela Bio, and MedImmune Ltd.

\section{Disclosure}

J. Beekman is an employee of Ipsos Public Affairs, a research firm paid to conduct this research study. A. Keisler was an employee of Ipsos Public Affairs, a research firm paid to conduct this research study. O. Pedraza is an employee of Ipsos Public Affairs, a research firm paid to conduct this research study. M. Haramura is an employee of Chugai Pharmaceutical Co., Ltd., which is conducting clinical trials focused on NMOSD and a sponsor of the current study. 
A. Gianella-Borradori was an employee of Chugai Pharmaceutical Co., Ltd., which is conducting clinical trials focused on NMOSD and a sponsor of the current study. E. Katz and J.N. Ratchford are employees of Viela Bio, which is conducting a clinical trial focused on NMOSD and a sponsor of the current study. G. Barron is an employee of MedImmune, which is conducting a clinical trial focused on NMOSD and a sponsor of the current study. L.J. Cook and J.M. Behne are supported in part by The Guthy-Jackson Charitable Foundation, which is a sponsor of the current study. T.F. Blaschke, T.J. Smith, and M.R. Yeaman are advisors to The GuthyJackson Charitable Foundation, which is a sponsor of the current study. Go to Neurology.org/NN for full disclosures.

\section{Publication history}

Received by Neurology: Neuroimmunology \& Neuroinflammation January 11, 2019. Accepted in final form April 8, 2019.

Appendix Authors

\begin{tabular}{|c|c|c|c|c|}
\hline Name & Location & Role & Contributions & Disclosure(s) \\
\hline $\begin{array}{l}\text { Jennine } \\
\text { Beekman, } \\
\text { PhD }\end{array}$ & $\begin{array}{l}\text { Ipsos Public } \\
\text { Affairs, } \\
\text { Washington, } \\
\text { DC }\end{array}$ & Author & $\begin{array}{l}\text { Designed/ } \\
\text { conceptualized } \\
\text { the study; } \\
\text { acquired the data; } \\
\text { analyzed the data; } \\
\text { interpreted the } \\
\text { data; and drafted } \\
\text { the manuscript } \\
\text { for intellectual } \\
\text { content. }\end{array}$ & $\begin{array}{l}\text { Dr. Beekman is } \\
\text { an employee of } \\
\text { Ipsos Public } \\
\text { Affairs, a research } \\
\text { firm paid to } \\
\text { participate in this } \\
\text { research study. }\end{array}$ \\
\hline $\begin{array}{l}\text { Aysha } \\
\text { Keisler, } \\
\text { PhD }\end{array}$ & $\begin{array}{l}\text { Ipsos Public } \\
\text { Affairs, } \\
\text { Washington, } \\
\text { DC }\end{array}$ & Author & $\begin{array}{l}\text { Designed/ } \\
\text { conceptualized } \\
\text { the study; } \\
\text { acquired the } \\
\text { data; analyzed } \\
\text { the data; } \\
\text { interpreted the } \\
\text { data; and drafted } \\
\text { the manuscript } \\
\text { for intellectual } \\
\text { content. }\end{array}$ & $\begin{array}{l}\text { Dr. Keisler was an } \\
\text { employee of } \\
\text { Ipsos Public } \\
\text { Affairs, a research } \\
\text { firm paid to } \\
\text { participate in this } \\
\text { research study. }\end{array}$ \\
\hline $\begin{array}{l}\text { Omar } \\
\text { Pedraza, } \\
\text { MPH }\end{array}$ & $\begin{array}{l}\text { Ipsos Public } \\
\text { Affairs, } \\
\text { Washington, } \\
\text { DC }\end{array}$ & Author & $\begin{array}{l}\text { Designed/ } \\
\text { conceptualized } \\
\text { the study; } \\
\text { acquired the data; } \\
\text { analyzed the data; } \\
\text { interpreted the } \\
\text { data; and drafted } \\
\text { the manuscript } \\
\text { for intellectual } \\
\text { content. }\end{array}$ & $\begin{array}{l}\text { Dr. Pedraza is an } \\
\text { employee of } \\
\text { Ipsos Public } \\
\text { Affairs, a research } \\
\text { firm paid to } \\
\text { participate in this } \\
\text { research study. }\end{array}$ \\
\hline $\begin{array}{l}\text { Masayuki } \\
\text { Haramura, } \\
\text { PhD }\end{array}$ & $\begin{array}{l}\text { Chugai } \\
\text { Pharmaceutical } \\
\text { Co., Ltd. }\end{array}$ & Author & $\begin{array}{l}\text { Designed/ } \\
\text { conceptualized the } \\
\text { study; interpreted } \\
\text { the data; and } \\
\text { reviewed and } \\
\text { revised the } \\
\text { manuscript for } \\
\text { intellectual } \\
\text { content. }\end{array}$ & $\begin{array}{l}\text { Dr. Haramura is } \\
\text { an employee of } \\
\text { Chugai } \\
\text { Pharmaceutical } \\
\text { Co., Ltd., which is } \\
\text { conducting } \\
\text { clinical trials } \\
\text { focused on } \\
\text { NMOSD. }\end{array}$ \\
\hline $\begin{array}{l}\text { Athos } \\
\text { Gianella- } \\
\text { Borradori, } \\
\text { MD }\end{array}$ & $\begin{array}{l}\text { Chugai Pharma } \\
\text { USA, Inc. }\end{array}$ & Author & $\begin{array}{l}\text { Designed/ } \\
\text { conceptualized } \\
\text { the study; } \\
\text { interpreted the } \\
\text { data; and } \\
\text { reviewed and } \\
\text { revised the } \\
\text { manuscript for } \\
\text { intellectual } \\
\text { content. }\end{array}$ & $\begin{array}{l}\text { Dr. Gianella- } \\
\text { Borradori was an } \\
\text { employee of } \\
\text { Chugai } \\
\text { Pharmaceutical } \\
\text { Co., Ltd., which is } \\
\text { conducting clinical } \\
\text { trials focused on } \\
\text { NMOSD and } \\
\text { a sponsor of this } \\
\text { study. }\end{array}$ \\
\hline
\end{tabular}

Appendix (continued)

\begin{tabular}{|c|c|c|c|c|}
\hline Name & Location & Role & Contributions & Disclosure(s) \\
\hline $\begin{array}{l}\text { Eliezer } \\
\text { Katz, MD }\end{array}$ & Viela Bio & Author & $\begin{array}{l}\text { Designed/ } \\
\text { conceptualized } \\
\text { the study; } \\
\text { interpreted the } \\
\text { data; and } \\
\text { reviewed and } \\
\text { revised the } \\
\text { manuscript for } \\
\text { intellectual } \\
\text { content. }\end{array}$ & $\begin{array}{l}\text { Dr. Katz is an } \\
\text { employee of Viela } \\
\text { Bio, which is } \\
\text { conducting } \\
\text { a clinical trial } \\
\text { focused on } \\
\text { NMOSD and } \\
\text { a sponsor of this } \\
\text { study. }\end{array}$ \\
\hline
\end{tabular}

\begin{tabular}{|c|c|c|c|c|}
\hline $\begin{array}{l}\text { John N. } \\
\text { Ratchford, } \\
\text { MD }\end{array}$ & Viela Bio & Author & $\begin{array}{l}\text { Designed/ } \\
\text { conceptualized } \\
\text { the study; } \\
\text { interpreted the } \\
\text { data; and } \\
\text { reviewed and } \\
\text { revised the } \\
\text { manuscript for } \\
\text { intellectual } \\
\text { content. }\end{array}$ & $\begin{array}{l}\text { Dr. Ratchford is } \\
\text { an employee of } \\
\text { Viela Bio, which is } \\
\text { conducting } \\
\text { a clinical trial } \\
\text { focused on } \\
\text { NMOSD and } \\
\text { a sponsor of this } \\
\text { study. }\end{array}$ \\
\hline
\end{tabular}

\begin{tabular}{|c|c|c|c|c|}
\hline $\begin{array}{l}\text { Gerard } \\
\text { Barron, } \\
\text { BSc (Hons) }\end{array}$ & Viela Bio & Author & $\begin{array}{l}\text { Designed/ } \\
\text { conceptualized } \\
\text { the study; } \\
\text { interpreted the } \\
\text { data; and } \\
\text { reviewed and } \\
\text { revised the } \\
\text { manuscript for } \\
\text { intellectual } \\
\text { content. }\end{array}$ & $\begin{array}{l}\text { Mr. Barron is an } \\
\text { employee of } \\
\text { Medlmmune, } \\
\text { which is } \\
\text { conducting } \\
\text { a clinical trial } \\
\text { focused on } \\
\text { NMOSD and } \\
\text { a sponsor of this } \\
\text { study. }\end{array}$ \\
\hline
\end{tabular}

Lawrence University of Author Designed/ Dr. Cook is

J. Cook, Utah, Salt Lake conceptualized supported in-part
PhD, MStat City, UT the study; acquired the Jackson data; analyzed Charitable the data; Foundation, interpreted the which is data; and a sponsor of this reviewed/revised research. the manuscript for intellectual content.

\begin{tabular}{|c|c|c|c|c|}
\hline $\begin{array}{l}\text { Jacinta M. } \\
\text { Behne, MS }\end{array}$ & $\begin{array}{l}\text { Guthy-Jackson } \\
\text { Charitable } \\
\text { Foundation, } \\
\text { Beverly Hills, CA }\end{array}$ & Author & $\begin{array}{l}\text { Designed/ } \\
\text { conceptualized } \\
\text { the study; } \\
\text { interpreted the } \\
\text { data; and revised } \\
\text { the manuscript } \\
\text { for intellectual } \\
\text { content. }\end{array}$ & $\begin{array}{l}\text { Ms. Behne is } \\
\text { supported in-part } \\
\text { by The Guthy- } \\
\text { Jackson } \\
\text { Charitable } \\
\text { Foundation, } \\
\text { which is } \\
\text { a sponsor of this } \\
\text { research. }\end{array}$ \\
\hline
\end{tabular}

\begin{tabular}{|c|c|c|c|c|}
\hline $\begin{array}{l}\text { Terrence F. } \\
\text { Blaschke, } \\
\text { MD }\end{array}$ & $\begin{array}{l}\text { Stanford } \\
\text { University, Palo } \\
\text { Alto, CA }\end{array}$ & Author & $\begin{array}{l}\text { Designed/ } \\
\text { conceptualized } \\
\text { the study; } \\
\text { interpreted the } \\
\text { data; and revised } \\
\text { the manuscript } \\
\text { for intellectual } \\
\text { content. }\end{array}$ & $\begin{array}{l}\text { Dr. Blaschke is an } \\
\text { Advisor to The } \\
\text { Guthy-Jackson } \\
\text { Charitable } \\
\text { Foundation, } \\
\text { which is } \\
\text { a sponsor of this } \\
\text { research. }\end{array}$ \\
\hline
\end{tabular}

Terry J. University of Author Designed/ Dr. Smith is an

Smith, MD Michigan, Ann Author conceptualized Advisor to The the study; $\quad$ Guthy-Jackson analyzed the Charitable data; interpreted Foundation,

the data; and which is drafted the a sponsor of this manuscript for research. intellectual content.

\begin{tabular}{|c|c|c|c|c|}
\hline $\begin{array}{l}\text { Michael R. } \\
\text { Yeaman, } \\
\text { PhD }\end{array}$ & $\begin{array}{l}\text { University of } \\
\text { California, los } \\
\text { Angeles, CA }\end{array}$ & Author & $\begin{array}{l}\text { Designed/ } \\
\text { conceptualized } \\
\text { the study; } \\
\text { analyzed the } \\
\text { data; interpreted } \\
\text { the data; and } \\
\text { drafted the } \\
\text { manuscript for } \\
\text { intellectual } \\
\text { content. }\end{array}$ & $\begin{array}{l}\text { Dr. Yeaman is an } \\
\text { Advisor to The } \\
\text { Guthy-Jackson } \\
\text { Charitable } \\
\text { Foundation, } \\
\text { which is } \\
\text { a sponsor of this } \\
\text { research. }\end{array}$ \\
\hline
\end{tabular}




\section{References}

1. Weinshenker BG, Wingerchuk DM. Neuromyelitis spectrum disorders. Mayo Clin Proc 2017;92:663-679.

2. Akaishi T, Nakashima I, Sato DK, et al. Neuromyelitis optica spectrum disorders. Neuroimaging Clin N Am 2017;27:251-265.

3. Marignier R, Cobo Calvo A, Vukusic S. Neuromyelitis optica and neuromyelitis optica spectrum disorders. Curr Opin Neurol 2017;30:208-215.

4. Whittam D, Wilson M, Hamid S, et al. What's new in neuromyelitis optica? A short review for the clinical neurologist. J Neurol 2017;264:2330-2344.

5. Flanagan EP, Cabre P, Weinshenker BG, et al. Epidemiology of aquaporin-4 autoimmunity and neuromyelitis optica spectrum. Ann Neurol 2016;79:775-783.

6. Houzen H, Kondo K, Niino M, et al. Prevalence and clinical features of neuromyelitis optica spectrum disorders in northern Japan. Neurology 2017;89:1995-2001.

7. Sepulveda M, Aldea M, Escudero D, et al. Epidemiology of NMOSD in Catalonia: influence of the new 2015 criteria in incidence and prevalence estimates. Mult Scler Epub 2017 Oct 1.

8. Pandit L, Asgari N, Apiwattanakul M, et al. Demographic and clinical features of neuromyelitis optica: a review. Mult Scler 2015;21:845-853.

9. Bukhari W, Prain KM, Waters P, et al. Incidence and prevalence of NMOSD in Australia and New Zealand. J Neurosurg Psych 2017;88:632-638.

10. Papp V, Illes Z, Magyari M, et al. Nationwide prevalence and incidence study of neuromyelitis optica spectrum disorder in Denmark. Neurology 2018;10:1212.

11. Pittock SJ, Lucchinett CF. Neuromyelitis optica and the evolving spectrum of autoimmune aquaporin-4 channelopathies: a decade later. Ann NY Acad Sci;1366:20-39.

12. Kessler RA, Mealy MA, Jimenez-Arango JA, et al. Anti-aquaporin-4 titer is not predictive of disease course in neuromyelitis optica spectrum disorder: a multicenter cohort study. Mult Scler Relat Disord 2017;17:198-201.

13. Zamvil SS, Slavin AJ. Does MOG Ig-positive AQP4-seronegative opticospinal inflammatory disease justify a diagnosis of NMO spectrum disorder ? Neurol Neuroimmunol Neuroinflamm 2015;2:e62. doi:10.1212/NXI0000000000000062.

14. Jarius S, Ruprecht K, Kleiter I, et al. MOG-IgG in NMO and related disorders: a multicenter study of 50 patients. Part 1: frequency, syndrome specificity, influence of disease activity, long-term course, association with AQP4-IgG, and origin. J Neuroinflamm 2016;13:279.

15. Alexion Pharmaceuticals. Alexion announces successful phase 3 PREVENT study of Soiris (Eculizumab) in patients with neuromyelitis optica spectrum disorders (NMOSD). Available at: alexionpharma.com/press-release. Accessed March 25, 2019. Also see ClinicalTrials.gov NCT01892345.

16. Chugai Pharmaceutical Co., LTD. Chugai presents results from phase III study of satralizumab in NMOSD at ECTRIMS 2018. Available at: chugai-pharm.co.jp. Accessed March 25, 2019. Also see ClinicalTrials.gov NCT02028884.

17. VielaBio Inc. A double-masked, placebo-controlled study with open label period to evaluate MEDI-551 in neuromyelitis optica and neuromyelitis optica spectrum disorder. Available at: ClinicalTrials.gov. NCT02200770. Accessed March 25, 2019.

18. Bradl M, Reindl M, Lassmann H. Mechanisms for lesion localization in neuromyeltis optica spectrum disorders. Curr Op Neurol 2018;31:325-333.

19. Papadopoulos MC, Bennett JL, Verkman AS. Treatment of neuromyelitis optica: state-of-the-art and emerging therapies. Nat Rev Neurol 2014;10:493-506.

20. Sato DK, Callegaro D, Lana-Peixoto MA, et al. Seronegative neuromyelitis optica spectrum - the challenges on disease definition and pathogensis. Arq Neuropsiq 2014;72:445-450.

21. Kim SH, Kim HJ. A step forward towards personalized immunosuppressive therapy in neuromyelitis optica spectrum disorder. J Neurol Neurosurg Psych 2017;88:619.

22. Moore P, Jackson C, Mutch K, et al. Patient-reported outcome measure for neuromyelitis optica: pretesting of preliminary instrument and protocol for further development in accordance with international guidelines. BMJ Open 2016;6:e011142.

23. Eaneff S, Wang V, Hanger M, et al. Patient perspectives on neuromyelitis optica spectrum disorders: data from the PatientsLikeMe online community. Mult Scler Relat Disord 2017;17:116-122.

24. Schmidt F, Zimmermann H, Mikolajczak J, et al. Severe structural and functional visual system damage leads to profound loss of vision-related quality of life in patients with neuromyelitis optica spectrum disorders. Mult Scler Relat Disord 2017;11: 45-50.
25. Shi Z, Chen H, Lian Z, et al. Factors that impact health-related quality of life in neuromyelitis optica spectrum disorder: anxiety, disability, fatigue and depression. J Neuroimmunol 2016;293:54-58.

26. Ware JE, Sherbourne CD. The MOS 36-item Short-Form Health Survey (SF-36): I. Conceptual framework and item selection. Med Care 1992;30:473-483.

27. Vickrey BG, Hays RD, Harooni R, et al. A health-related quality of life measure for multiple sclerosis. Qual Life Res 1995;4:187-206.

28. Weih LM, Hassell JB, Keeffe JE. Assessment of the impact of vision impairment. Invest Ophthal Vis Sci 2002;43:927-935.

29. Lionbridge Technologies, Inc. Translation Workspace XLiff editor, version 2.49.1. Waltham:Lionbridge; 2018. Available at: Lionbridge.com. Accessed March 25, 2019.

30. Wingerchuk DM, Lennon VA, Pittock SJ, et al. Revised diagnostic criteria for neuromyelitis optica. Neurology 2006;66:1485-1489.

31. Wingerchuk DM, Banwell B, Bennett JL, et al. International consensus diagnostic criteria for neuromyelitis optica spectrum disorders. Neurology 2015;85: 177-189.

32. IBM SPSS Statistics Software, Version 25.0. Armonk: IBM Corporation; 2017. Available at: IBM SPSS Software. Accessed March 25, 2019.

33. Georgopoulou S, Efraimidou S, MacLennan SJ, et al. Antiphospholipid (Hughes) syndrome: description of population and health-related quality of life (HRQoL) using the SF-36. Lupus 2015;24:174-179.

34. Jenkinson C, Fitzpatrick R, Swash M, et al. The ALS Health Profile Study: quality of life of amyotrophic lateral sclerosis patients and careers in Europe. J Neurol 2000;247: $835-840$.

35. Riazi A, Hobart JC, Lamping DL, et al. Using the SF-36 measure to compare the health impact of multiple sclerosis and Parkinson's disease with normal population health profiles. J Neurol Neurosurg Psych 2003;74:710-714.

36. Wolfe F, Michaud K, Li T, et al. EQ-SD and SF-36 quality of life measures in systemic lupus erythematosus: comparisons with rheumatoid arthritis, non-inflammatory rheumatic disorders and fibromyalgia. J Rheum 2010;37:296-304.

37. Jenkinson C, Hobar J, Chandola T, et al. Use of the short form health survey (SF-36) in patients with amyotrophic lateral sclerosis: tests of data quality, score reliability, response rate and scaling assumptions. J Neurol 2002;249:178-183.

38. Ong AD, Bergeman CS, Bisconti TL, et al. Psychological resilience, positive emotions, and successful adaptation to stress in later life. J Personal Soc Psych 2006;91:730-749.

39. Tugade MM, Fredrickson BL, Feldman-Barrett L. Psychological resilience and positive emotional granularity: examining the benefits of positive emotions on coping and health. J Pers 2004;72:1161-1190.

40. Borisow N, Mori M, Kuwabara S, et al. Diagnosis and treatment of NMO spectrum disorder and MOG-encephalitis. Front Neurol 2018;9:888.

41. Hyun JW, Jeong IH, Joung A, et al. Evaluation of the 2015 diagnostic criteria for neuromyelitis optica spectrum disorder. Neurology 2016;86:1772-1779.

42. Zhao S, Mutch K, Elsone L, et al. Neuropathic pain in neuromyelitis optica affects activities of daily living and quality of life. Mult Scler J 2014;20:1658-1661.

43. Sakakibara R. Neurogenic lower urinary tract dysfunction in multiple sclerosis, neuromyelitis optica and related disorders. Clin Auton Res Epub 2018 Aug 3.

44. Methley AM, Mutch K, Moore P, et al. Development of a patient-centered conceptual framework of health-related quality of life in neuromyelitis optica: a qualitative study. Health Expect 2017;20:47-58.

45. Mutch K, Zhao S, Hamid S, et al. Bladder and bowel dysfunction affect quality of life: a cross sectional study of 60 patients with aquaporin- 4 antibody-positive neuromyelitis optica spectrum disorder. Mult Scler Relat Disord 2015;4: 614-618.

46. Chanson J-B, Zephir H, Collongues N, et al. Evaluation of health-related quality of life, fatigue and depression in neuromyelitis optica. Eur J Neurol 2011;18:836-841.

47. Chavarro VS, Mealy MA, Simpson A, et al. Insufficient treatment of severe depression in neuromyelitis optica spectrum disorder. Neurol Neuroimmunol Neuroinflamm 2016;3:e286. doi:10.1212/NXI.0000000000000286.

48. Yeaman MR, Jackson V. Rare to the Rescue. The Scientist. 2018. Available at: thescientist.com. Accessed March 25, 2019.

49. Jackson V, Yeaman MR. The Power of Rare. New York: Simon \& Schuster; 2017. Available at: simonandschuster.com/The-Power-of-Rare. Accessed March 25, 2019. 


\section{Neurology \\ Neuroimmunology \& Neuroinflammation}

\section{Neuromyelitis optica spectrum disorder: Patient experience and quality of life Janine Beekman, Aysha Keisler, Omar Pedraza, et al. \\ Neurol Neuroimmunol Neuroinflamm 2019;6; \\ DOI 10.1212/NXI.0000000000000580}

This information is current as of June 20, 2019

\section{Updated Information \& Services}

References

Citations

Subspecialty Collections

Permissions \& Licensing

Reprints including high resolution figures, can be found at: http://nn.neurology.org/content/6/4/e580.full.html

This article cites 39 articles, 5 of which you can access for free at: http://nn.neurology.org/content/6/4/e580.full.html\#\#ref-list-1

This article has been cited by 4 HighWire-hosted articles: http://nn.neurology.org/content/6/4/e580.full.html\#\#otherarticles

This article, along with others on similar topics, appears in the following collection(s):

Autoimmune diseases

http://nn.neurology.org//cgi/collection/autoimmune_diseases Cohort studies

http://nn.neurology.org//cgi/collection/cohort_studies Quality of life

http://nn.neurology.org//cgi/collection/quality_of_life

Information about reproducing this article in parts (figures,tables) or in its entirety can be found online at:

http://nn.neurology.org/misc/about.xhtml\#permissions

Information about ordering reprints can be found online: http://nn.neurology.org/misc/addir.xhtml\#reprintsus

Neurol Neuroimmunol Neuroinflamm is an official journal of the American Academy of Neurology.

Published since April 2014, it is an open-access, online-only, continuous publication journal. Copyright Copyright () 2019 The Author(s). Published by Wolters Kluwer Health, Inc. on behalf of the American Academy of Neurology.. All rights reserved. Online ISSN: 2332-7812.

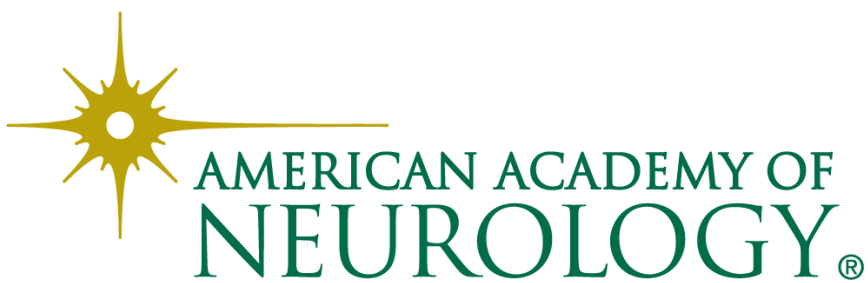

